\title{
SVM Classifier for Impulse Fault Identification in Transformers using Fractal Features
}

\author{
Chiranjib Koley \\ Haldia Institute of Technology, Haldia \\ Midnapur (E), West Bengal, 721657, India \\ Prithwiraj Purkait \\ Haldia Institute of Technology, Haldia \\ Midnapur (E), West Bengal, 721657, India \\ and Sivaji Chakravorti \\ High Voltage Laboratory, Electrical Engineering Department, \\ Jadavpur University, Kolkata, West Bengal, India,
}

\begin{abstract}
Improper or inadequate insulation may lead to failure during impulse tests of a transformer. It is important to identify the type and the exact location of insulation failure within the winding of power transformers. This paper describes a new approach using fractal theory for extraction of features from the impulse test response of a transformer and Support Vector Machine (SVM) in regression mode to classify the fault response patterns. A variety of algorithms are available for the computation of Fractal Dimension (FD). In the present work, Box counting and Higuchi's algorithm for the determination of FD, Lacunarity, and Approximate Entropy (ApEn) has been used for the extraction of fractal features form time domain impulse test response. The analysis has been performed on both Analog and Digital Models of a 3 MVA, 33/11 kV transformer. A noticeable finding is that the SVM tool trained with the simulated data only is capable of identifying the location and fault classes of analog model data accurately within a tolerance limit of $\pm 3.37 \%$.

Index Terms - Analog model, approximate entropy, digital model, Fractal dimension, impulse fault identification, Lacunarity, support vector machine, transformer fault analysis.
\end{abstract}

\section{INTRODUCTION}

TRANSFORMER windings are often exposed to different short-duration impulse voltage of high amplitude produced by lightning or switching transients, and by insulator flash over. These impulses may cause permanent damage to the insulation of the winding. In order assess the withstand capability of insulation, transformers are impulse tested in laboratory as per standard [1]. Fault may occur in the winding during such tests due to improper or inadequate insulation. In the case of such a fault, it is essential to detect the fault and its location in the winding for taking necessary corrective measures. In the case of a fault within the winding, the shape of the winding current changes as compared to that of a healthy winding. It has been established that the pattern of fault currents contain a typical signature of type and location of the fault for a

Manuscript received on 24 May 2006, in final form 20 February 2007. given winding [2]. It is of great practical importance to asses the type of fault and also to identify exact location of fault during such tests. All the faults involving non self-restoring insulation are destructive in nature and hence have to be necessarily rectified. In the case of failure of a transformer during an impulse test, manufacturers have to detank the winding in order to locate the fault. It would be appropriate to develop a method for accurate fault location so that results are available prior to the detanking the transformer.

For many years, the applied voltage waveforms and the resulting current waveforms were analyzed manually in time domain by studying oscillographic records [2]. But this technique was strongly dependent on the knowledge and experience of the experts performing the analysis. With the advent of digital recorders and analyzers, frequency domain based analysis became popular, particularly the transfer function approach [3], for fault 
classification. In recent years, the use of computer aided techniques like Expert Systems [4], and Artificial Neural Network s(ANN) [5] have been employed for impulse fault classification in transformers. Very recently, due to non-stationary nature of the impulse response Wavelet analysis [6] i.e., time-frequency domain based analysis, has also been employed for impulse fault classification in transformers.

Studies on impulse fault diagnosis using the above mentioned methods showed that localization of faults could be done within $25 \%$ of the winding length. Because of the complex nature of composite insulation system of transformers, determination of exact location of faults within transformer windings is a difficult task in many practical cases. In recent past researchers [7] have applied Fuzzy ARTMAP on frequency response data to localize power transformer faults. They found that localization of faults can be done within $20 \%$ of the winding.

Presently there is an increasing trend on the application of fractal techniques in the field of signal processing and pattern recognition, as it has solved many pattern recognition problems efficiently [8]. Fractal techniques has also been applied for partial discharge pattern recognition [9] and also in the field of classifying impulse faults of transformers [10]. Researchers in [10] have successfully classified type and location of fault within $33 \%$ of transformer winding with the help of two Fractal features, viz. FD using box count algorithm, and Lacunarity.

In the present paper, Fractal features have been extracted from time domain impulse response of transformer winding. The Fractal features include calculation of FD using box count and Higuchi algorithm, Lacunarity and ApEn, as these features were found to provide best clarity in results.

SVM [11] in recent years has proved to be an advanced tool in solving classification or pattern recognition problems. SVM surpassed other classification schemes, including advanced statistical decision methods, as accurate classifications could be achieved with small number of support vectors with the consequent benefit in computational cost [12]. The present paper is aimed at applying SVM for classifying fractal features of fault responses, so that static as well as dynamic impulse faults in transformers could be identified and localized with greater degree of accuracy.

The present study has been based on static and dynamic impulse faults simulated in Electro Magnetic Transient Program (EMTP) based digital model of a 3 MVA, 33/11 kV transformer and an analog model of $3 \mathrm{MVA}, 33 / 11 \mathrm{kV}, 3-$ phase, $50 \mathrm{~Hz}$, ON, Dy11 transformer.

\section{DATA GENERATION FROM ANALOG AND DIGITAL MODELS OF 3 MVA TRANSFORMER}

The data for the task of impulse fault identification as reported in this paper have been acquisitioned/ generated with the help of analog as well as time-frequency domain based digital model of a transformer winding. In both analog and digital models, the impulse current through the winding have been determined on the basis of an incident lightning impulse wave of standard $1.2 / 50 \mu s$ waveshape. Impulse current waveforms had been recorded following the tank current method [14].

\subsection{DATA ACQUISITION FROM ANALOG MODEL}

An analog model has been constructed which is based on design data of a real-life 3 MVA, 33/11 kV, 3-phase, $50 \mathrm{~Hz}$, Dy11 transformer. HV winding of the transformer is considered to be impulse tested as per IEC publication 60076-4, 2002 [1]. Impulse data acquisition set-up incorporating the analog model is shown in Figure 1.

The impulse voltage and winding current waveforms for both reduced (50\% BIL) impulse and full (100\% BIL) impulse waves were recorded by a Tektronix TDS 320, $100 \mathrm{MHz}, 500 \mathrm{MS} / \mathrm{s}$ digital storage oscilloscope. The waveforms were then transferred to the PC through an RS-232 interface. "Wave Star" software was used in the PC for acquiring data from the oscilloscope. The waveforms were subsequently transferred to the datastorage of system. Details of the analog model and experimental setup can be found in [4].

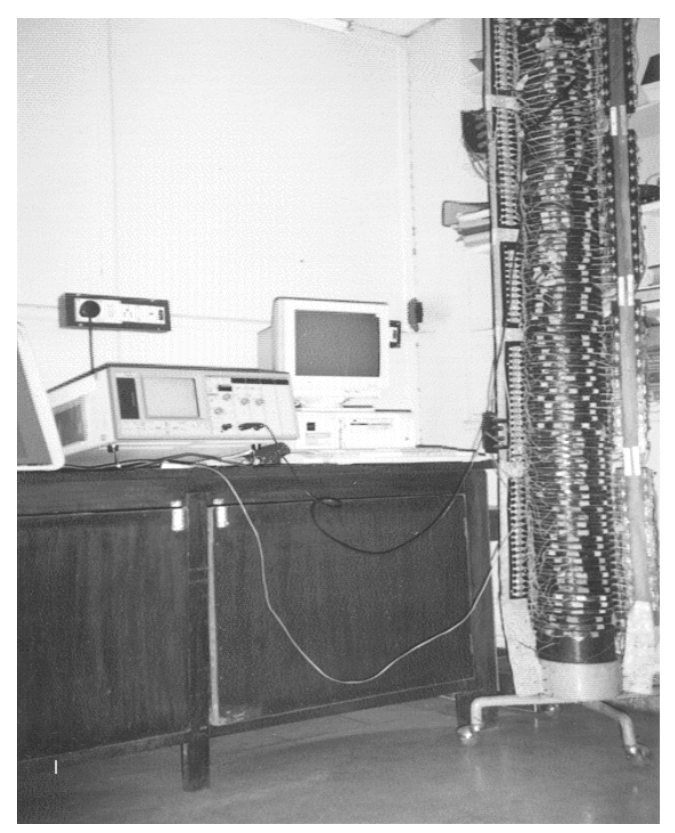

Figure 1. Test set-up incorporating the analog model.

\subsection{DATA GENERATION FROM DIGITAL MODEL}

For fault simulation and data generation, a time-frequency domain based high frequency lumped parameter digital model of the $33 \mathrm{kV}$ winding of a 3 MVA, 33/11 kV transformer has been prepared, in EMTP. The constructed digital model is shown in Figure 2. 


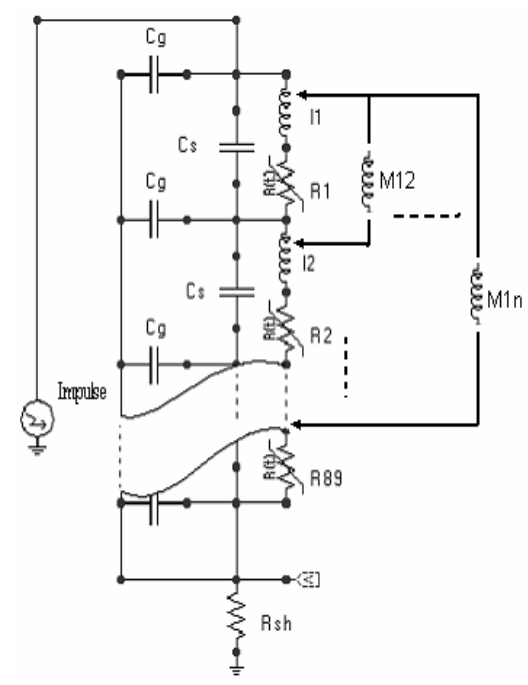

Figure 2. Lumped parameter EMTP model of 3 MVA Transformer.

In the present paper, circular coils of rectangular stacked section have been used for parameter calculation of the disk winding used in the transformer. The formulae for calculation of inductance (l), series capacitance (Cs) and ground capacitance $(\mathrm{Cg})$, and resistance $(\mathrm{R})$ are available in [14].

\subsection{DE-NOISING OF ANALOG DATA}

Unlike the simulated impulse current responses obtained from the EMTP model of 3 MVA transformer, the impulse current responses obtained from the analog model of a 3 MVA transformer under different fault conditions contain noise. So these impulse current signals obtained from the analog model need to be filtered to reduce noise prior to further processing of the signal.

In order to denoise these signals, a wavelet packet based filter has been used. The Stein's Unbiased Estimate of Risk (SURE) [15] principle has been chosen for setting the threshold (T) for de-noising of the impulse current signals. The detailed discussion on the filter can be found in [16]. Figure 3 shows a noisy and de-noised impulse current signal obtained under no-fault condition. The two signals have been plotted with a vertical shift for better clarity.

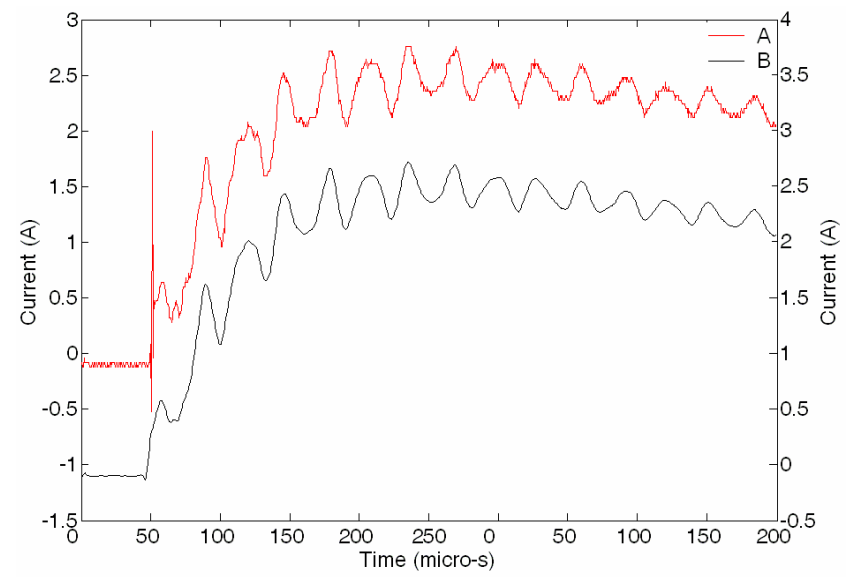

Figure 3. (A) Signal obtained from the analog model transformer. (B) Denoised signal.

\section{SIMULATION OF STATIC AND DYNAMIC FAULTS}

For the simulation of faults a time-frequency domain based high frequency lumped parameter digital model of the $33 \mathrm{kV}$ winding of a 3 MVA, 33/11 $\mathrm{kV}$ transformer has been prepared, as shown in Figure 2. The accuracy of the timefrequency domain based digital model has been verified by comparing the impulse test responses of 3 MVA, 30 MVA and 104 MVA real life transformers and corresponding timefrequency domain based digital models of those transformers. Details of the modeling technique and obtained results have been discussed in [13]. Good agreement between the results obtained from digital models and corresponding real life tests of such a wide range of transformers, as reported in [13], demonstrates the accuracy of the digital models. To further demonstrate the accuracy of the digital modeling technique used in the work, a comparative study has been done on the neutral current response during impulse test on a $220 / 110 \mathrm{kV}$ transformer. The following Figure 4 shows such currents corresponding to the digital model transformer and also the current record reported in IEC 60076-4-2002 [1] for transformers of the above-mentioned rating. Reasonably good agreement between the two current records indicates the degree of accuracy of the developed digital modeling technique. Slight deviation between the two waveforms may be attributed to the fact that exact design data for the transformer has not been reported in the referred IEC, and hence the digital model is based on design data collected from data base and practical experiences.

Insulation failures may result in two classes of winding faults in a transformer during impulse tests - namely series and shunt faults. Both these two fault classes may, once again be of two types - static and dynamic. Static faults are those which are already present in the transformer winding when the impulse is applied and do not change their characteristics during the passage of impulse through the winding. Dynamic faults, on the other hand, occur during the passage of the impulse wave through the length of the winding and manifest itself dynamically during the impulse test. Series fault implies insulation failure between the discs or between the turns, while shunt fault represents insulation failure between the winding and earthed components like tank, core etc. Both these classes of faults may occur anywhere along the entire length of the winding.

\subsection{STATIC FAULT SIMULATION}

During static fault simulation, the faulted disc has been apriori short-circuited and connected to ground for series and shunt faults, respectively, and then impulse voltage has been applied. During the simulation of series fault, as the corresponding disc is short circuited, so no current flows through that disc. Hence the mutual inductance effect of the faulted disc on the other discs will be absent. The mutual inductance of other discs will also change accordingly. This situation has been incorporated in the digital model of the 
transformer during simulation of faults. For the digital model, faults have been simulated in each of the discs, and for the analog model faults have been simulated for alternate (odd numbered) discs. No-fault condition has been simulated four times to investigate the effects of possible variations in test conditions on the no-fault current waveform. Figure 5 shows sample plots of the transformer winding currents obtained by the tank-current method corresponding to a series fault at disc 45 for the analog and digital models of the 3 MVA transformer.

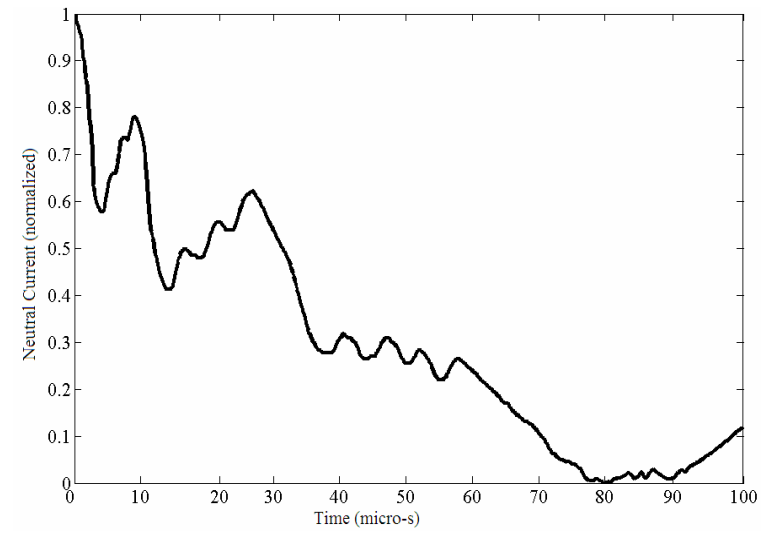

(A)

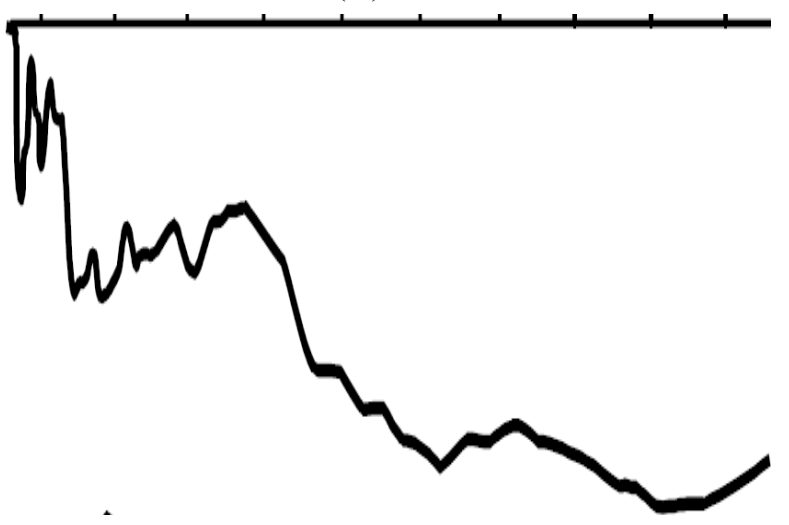

(B)

Figure 4. Neutral current response of 220/110 kV Transformer with $100 \mu \mathrm{s}$ sweep. (A) Simulated response of the $220 / 110 \mathrm{kV}$ digital model transformer (B) Original response of real life transformer $220 / 110 \mathrm{kV}$ [1].

\subsection{DYNAMIC FAULT SIMULATION}

During application of impulse voltage on transformer winding, series and shunt faults may develop within the transformer winding. Initiation of dynamic faults in the winding will take place with certain time delay depending on the mechanism of insulation breakdown. Depending upon the condition of oil paper composite insulation system and its moisture content, the time required for breakdown varies over a wide range. In order to simulate these types of faults, time dependent switch has been utilized with the time-frequency domain based digital model. Figure 6 shows the tank current response of dynamic and static series fault waveforms for 3 MVA transformer at disc number 30. As may be seen, there are notable differences in "static" and "dynamic" current waveforms corresponding to a fault.

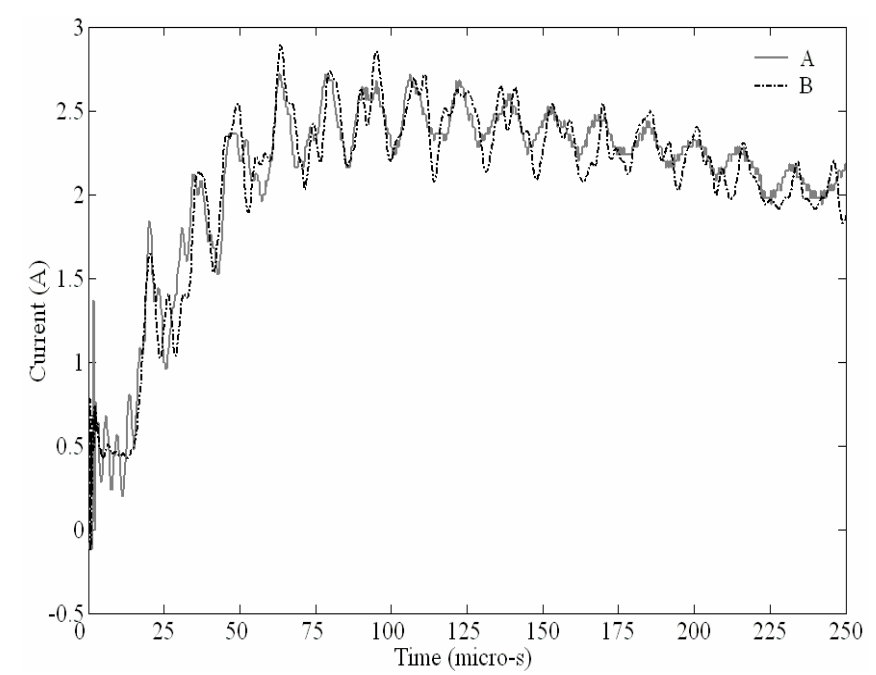

Figure 5. Tank current vs. Time: (A) Digital model (B) Analog model of 3 MVA transformer.

\section{FRACTAL THEORY}

Since the early work of Mandelbrot [17], the interest on fractal analysis has increased tremendously. Many natural forms and phenomena seem, in the view of their irregular character, suitable for being described as fractals. Indeed one might consider that any form can be characterized as a fractal whose description in terms of the primitives of Eucliden geometry is 'impractical'. The variability of this approach has been proved by numerous applications in Computer Graphics and Signal Processing.

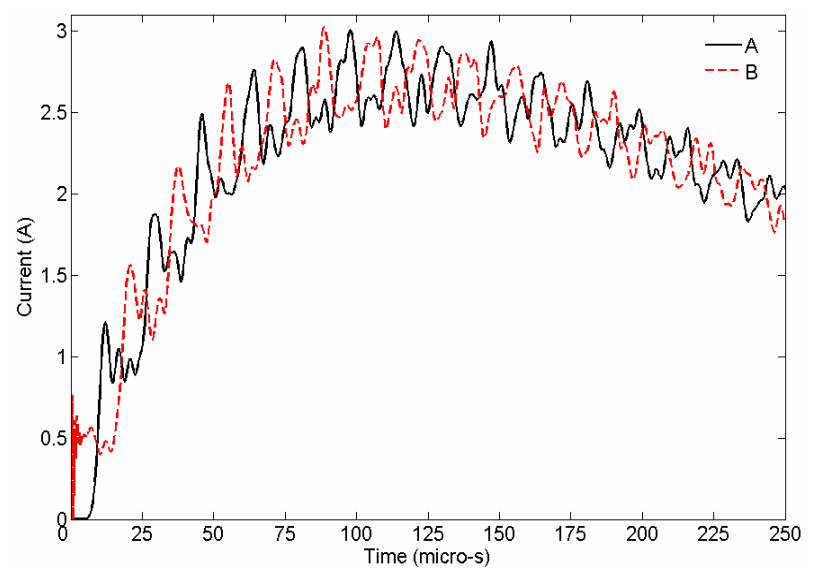

Figure 6. Tank current vs. Time: (A) Dynamic series fault occurs after $10 \mu \mathrm{s}$ from the application of impulse (B) Static series fault in the case of 3 MVA transformer at disc number 30 .

\subsection{FD BOX COUNT}

Fractal dimension is an important characteristic of fractals because it contains information about their geometric structure. The term fractal dimension was introduced by Mandelbrot [17]. According to him, a set A in an Euclidean n-space, is said to be self-similar when $A$ is the union of $\mathrm{N}$ distinct (non-overlapping) copies of itself, each of which has been scaled down by a ratio $r$ in all co- 
ordinates. The fractal dimension $\mathrm{D}$ of the set $\mathrm{A}$ is given by the relation [17]:

$$
\begin{aligned}
& N r^{D}=1 \\
& \text { or } \\
& D=\frac{\log N}{\log (1 / r)}
\end{aligned}
$$

While the definition of fractal dimension by self-similarity is straightforward, it is often difficult to estimate directly from the image data. However, a related measure of the fractal dimension, the box-dimension, can be more easily computed from a fractal set $\mathrm{A}$ in an n-dimensional space [10]. Let, the entire set $A$ is covered by a single $n$-dimensional box of size $L_{\max }$ which can be a multiple of the side length $L$ in the $\mathrm{x}-\mathrm{y}$ plane. If the set $\mathrm{A}$ is now scaled own by the ratio $\mathrm{r}$, then the number of boxes of size $L=r L_{\max }$ needed to cover the whole can be derived from (1) as:

$$
N(L)=\frac{1}{r^{D}}=\left[\frac{L_{\max }}{L}\right]^{D}
$$

The simplest way to estimate D from (2) is to divide the ndimensional space into a grid of boxes with side length $L$ (i.e. square of side $L$ for the present case of 2-dimensional space) and to count the number of non-empty boxes. If such a box contains at least one image point, we call it a non-empty box. For a fixed $L$, let the total number of non-empty boxes be $\mathrm{N}(L)$. Several values of $L$ are chosen and the corresponding $\mathrm{N}(\mathrm{L})$ are determined. Fractal dimension $\mathrm{D}$ is then obtained from the slope of a least square linear fit on the plot $[\log (L)$ $\log (\mathrm{N}(L))]$.

\subsection{FD HIGUCHI}

Higuchi's algorithm for the calculation of FD regarded as most stable estimator of FD [18].

Considering $x(1), x(2), x(3), \ldots, x(N)$ as the time sequence to be analyzed. Construct $k$ new time series $x_{m}^{k}$ as

$$
\begin{aligned}
& x_{m}^{k}=\{x(m), x(m+k), x(m+2 k), \ldots, \\
& \left.x\left(m+\left\lfloor\frac{N-m}{k}\right\rfloor k\right)\right\}, \quad \text { for } m=1,2, \ldots, k
\end{aligned}
$$

where $m$ indicates the initial time value, $k$ indicates the discrete time interval between points (delay), and $\lfloor a\rfloor$ means integer part of $a$. For each of the curves or time series $x_{m}^{k}$ constructed, the average length $L_{m}(k)$ is computed as

$$
L_{m}(k)=\frac{\left\lfloor\frac{N-m}{k}\right\rfloor|x(m+i k)-x(m+(i-1) k)|(n-1)}{\left\lfloor\frac{N-m}{k}\right\rfloor k}
$$

where $N$ is the total length of the data sequence $x$ and $(N-1) /\lfloor(N-m) / k\rfloor k$ is a normalization factor. An average length is computed for all time series having the same delay (or scale) $k$, as the mean of the $k$ lengths $L_{m}(k)$ for $m=1, \ldots, k$. This procedure is repeated for each $k$ ranging from 1 to $k_{\max }$, yielding an sum of average lengths $L(k)$ for each $k$ as indicated in equation (3)

$L(k)=\sum_{m=1}^{k} L_{m}(k)$

The total average length for scale $k, L(k)$, is proportional to $k^{-D}$, where $D$ is the FD by Higuchi's method. In the curve of $\ln (L(k))$ versus $\ln (1 / k)$, the slope of the least squares linear best fit is the estimate of the fractal dimension [18].

\subsection{LACUNARITY}

Among the various fractal features that can be computed from a complex pattern or texture, the fractal dimension is the primary one. However, it has been observed that fractal dimension alone is insufficient for the purpose of discrimination, since two differently appearing surfaces can have the same value of D. To overcome this, Mandelbrot [16] introduced the term called lacunarity $\lambda$, which quantifies the denseness of an image surface. Many definitions of this term have been proposed and the basic idea is to quantify the gaps or lacunae present in the given pattern.

A procedure for calculating Lacunarity is given in [19]. Let $\mathrm{P}(\mathrm{m}, \mathrm{L})$ be the possibility that there are $\mathrm{m}$ points within a box of side L (i.e. square of side L), which is centered about an arbitrary point on the waveform. Then $\mathrm{P}(\mathrm{m}, \mathrm{L})$ is normalized, as below for all $\mathrm{L}$

$\sum_{m=1}^{n} P(m, L)=1$

where $\mathrm{n}$ is the number of possible points within the box of side length $\mathrm{L}$. Let, the image is of size $\mathrm{L}_{\max } \times \mathrm{L}_{\max }$. If the entire waveform is overlayed with boxes of size $\mathrm{L}$, then the number of boxes with $\mathrm{m}$ points inside the box is $\left(\mathrm{L}_{\max } / \mathrm{m}\right) \mathrm{P}(\mathrm{m}, \mathrm{L})$.

Defining $G(L)$ and $G^{2}(L)$, Voss [19] defined lacunarity as:

$G(L)=\sum_{m=1}^{n} m P(m, L)$

$G^{2}(L)=\sum_{m=1}^{n} m^{2} P(m, L)$

$\lambda(L)=\frac{G^{2}(L)-[G(L)]^{2}}{[G(L)]^{2}}$ 


\subsection{APPROXIMATE ENTROPY}

Approximate Entropy (ApEn) is a "regularity statistic" that quantifies the unpredictability of fluctuations in a time series [20]. ApEn reflects the likelihood that "similar" patterns of observations will not be followed by additional "similar" observations. A time series containing many repetitive patterns has a relatively small ApEn; a less predictable (i.e., more complex) process has a higher ApEn [21].

The algorithm for computing ApEn has been published elsewhere [20]. Here a brief summary of the calculations, as applied to a time series of impulse current responses $\operatorname{IR}(i)$ of transformer under test has been provided. Given a sequence $S_{N}$, consisting of $N$ samples of impulse current response $\operatorname{IR}(1), \operatorname{IR}(2), \ldots ., \operatorname{IR}(N)$ the values of two input parameters, $m$ and $r$ has been chosen, to compute the approximate entropy, $\operatorname{ApEn}\left(S_{N}, m, r\right)$, of the sequence. The second of these parameters, $m$, specifies the pattern length, and the third, $r$, defines the criterion of similarity. Denoting a subsequence (or pattern) of $m$ samples, beginning at measurement $i$ within $S_{N}$, by the vector $p_{m}(i)$. Two patterns, $P_{m}(i)$ and $P(j)$, are similar if the difference between any pair of corresponding measurements in the patterns is less than $r$, i.e., $|\operatorname{IR}(i+k)-I R(j+k)|<r$ for $\quad 0 \leq k<m$

Now considering the set $P_{m}$ of all patterns of length $m$ [i.e., $\left.p_{m}(1), p_{m}(2), \ldots, p_{m}(N-m+1)\right]$, within $S_{N}$, define

$$
C_{i m}(r)=\frac{n_{i m}(r)}{N-m+1}
$$

where $n_{i m}(r)$ is the number of patterns in $P_{m}$ that are similar to $P_{m}(i)$ (given the similarity criterion $r$ ). The quantity $C_{i m}(r)$ is the fraction of patterns of length $m$ that resemble the pattern of the same length that begins at interval $i$. Now after calculating $C_{i m}(r)$ for each pattern in $P_{m}$, and defining $C_{i m}(r)$ as the mean of these $C_{i m}(r)$ values. The quantity $C_{i m}(r)$ expresses the prevalence of repetitive patterns of length $m$ in $S_{N}$. Finally, the approximate entropy of $S_{N}$, for patterns of length $m$ and similarity criterion $r$, is defined as.

$$
\operatorname{ApEn}\left(S_{N}, m, r\right)=\ln \left[\frac{C_{m}(r)}{C_{m+1}(r)}\right]
$$

\section{FAULT CHARACTERIZATION USING FRACTAL FEATURES}

Using the above mentioned techniques, fractal features have been extracted from the time domain impulse response of series fault, shunt fault and no-fault conditions for both analog and digital models of the transformer. Details of the signal acquisition/generation have been discussed in section 2. For each of the signal, FD using box count algorithm, FD using Higuchi's algorithm, Lacunarity and ApEn have been calculated. Figure 7 shows the variations of the FDs due to change in nature and location of fault for digital model as well as analog model of the transformer. It can be observed that the variations in the FD values obtained using Higuchi's algorithm is less for the series fault condition (when the location of fault changes), but for shunt fault condition the variation is almost linear. It can be also observed that the variation of FD obtained using box count algorithm is comparatively higher for series fault condition, and at the same time it is having higher deviation in the FD pattern between the digital model and analog model of the transformer. Figure 8 shows the variations of the ApEn and Lacunarity due to change in nature and location of fault for digital model as well as analog model of the transformer.

From the plots of Figure 7 and Figure 8 it can be observed that the feature vector pattern becomes more complex for analog model transformer in comparison with digital model of the transformer. It was noted that for proper identification of the location of fault it was necessary to obtain feature vector containing all the 4 features (FD box count; FD Higuchi; Lacunarity; ApEn). After constructing the feature vector for both analog and digital models of the transformer it was presented to Support Vector Machine (SVM) so that the type and location of the faults are identified.

\section{THEORY OF SVM}

The SVM technique, developed by Vapnik [22], was originally proposed essentially for classification problems of two classes but was found to be useful to deal with nonlinearly separable cases too. The details of relevant theory has been discussed in [16]. Here a brief discussion has been provided.

Real-world classification problems typically involve data that can only be separated using a nonlinear decision surface. Optimization on the input data in this case involves the use of a kernel-based transformation [23]: $\mathrm{K}$ is a kernel function such that for all input $x, z \in X$ where $\mathrm{x}, \mathrm{z}$ are input and $\mathrm{X}$ is input space, $\phi$ is a mapping from $\mathrm{X}$ to an (inner product) feature space $\mathrm{F}$.

$$
K(x, z)=\langle\phi(x) \bullet \phi(z)\rangle
$$

Kernels allow a dot product to be computed in a higher dimensional space without explicitly mapping the data into these spaces. A kernel-based decision function has the form [22]

$$
f(x)=\sum_{i=1}^{l} \alpha_{i} y_{i} K\left(x, x_{i}\right)+b
$$

where $\mathrm{b}$ is bias, $\mathrm{y}$ is output, $\alpha$ Lagrange multiplier, $l$ is training set size, and $\mathrm{I}$ is the number of training sets.

Radial basis function (RBF) kernels are extremely popular and often deliver better performance [11].

The RBF kernel has the form

$K(x, y)=\exp \left(-\gamma\|x-y\|^{2}\right)$

where $\gamma$ is a constant. 


\section{FAULT IDENTIFICATION}

\subsection{FAULT IDENTIFICATION FOR DIGITAL MODEL}

The data for SVM are the normalized values of the four parameters obtained from the digital model of the transformer, viz. FD box count, FD Higuchi, Lacunarity and ApEn, related to impulse response of transformer under series fault, shunt fault and no-fault conditions. Feature vectors have been organized in series fault, no-fault and shunt fault sequences. In the case of series fault the sequence start form the line end disc (i.e. disc 1) to earth end (i.e. disc 89) but in the case of shunt fault the sequence start from earth end to line end disc. Such type of organization of feature vectors is based on the topological similarity between the features, so that the classification problem becomes easier.

\subsection{TRAINING PROCEDURE AND SELECTION OF SVM PARAMETERS}

raining procedure and choice of SVM parameters for training is very important for classification. It uses the selected features of impulse current under different fault conditions at different fault locations. In this respect, two types of faults, have been considered, i.e. series and shunt fault, which may occur anywhere along the length of the winding as discussed previously. According to the fault type and fault location in a particular section of winding, a range of values have been assigned, which work as the target value range for that type and location of the fault. The assigned target value ranges are based on a properly chosen linear function i.e.

$$
T=-10 x+1830+R(5)
$$

where $T$ is the target value range and $\mathrm{x}$ is the sequence number of feature vectors and $R(5)$ is any random number between +5 to -5 .

Each fault represents a range of values instead of a single value. For example shunt faults in disc no 5 and 10 corresponds to the range 45 to 55 and 95 to 105 , respectively. This automatically takes into account any possible variations due to inherent inaccuracies of modeling, if any, within a limited band. For better understanding of the training process Table-1 have been given, which shows some of the representative faults and their range of target values. Such a range of target values for each type and location of faults will enable the SVM classifier to be tolerant to variations due to modeling inaccuracies to a certain extent.

After fixing the training procedure, different parameter values of the SVM classifier have been adjusted. In the current application, kernel such as dot, polynomial, radial and neural has been investigated, but the radial kernel with $\gamma=1.34$ has given better results than other kernels. The choices of other parameter values were based on trial and error.

\subsubsection{RESULTS AND OBSERVATIONS}

After training, feature vectors except those which were used for training were presented to SVM for prediction of fault classes. The results obtained after prediction of SVM have been plotted in Figure 9. If the predicted value of SVM fall in the target zone, then the SVM predicted faulted results are said to be correct. A close observation of Figure 9 shows that SVM is able to identify the fault nature and location correctly in most of the cases. The tolerance for location of fault is calculated to be as small as $\pm 1.12 \%$.

Table 1. Fault type and location with their feature sequence number and range of target values.

\begin{tabular}{cccc}
\hline \hline Disc Number & Fault Type & $\begin{array}{c}\text { Feature } \\
\text { sequence } \\
\text { number (x) }\end{array}$ & $\begin{array}{c}\text { Range of } \\
\text { Target value }\end{array}$ \\
\hline 1 & Shunt & 182 & $5-15$ \\
2 & Shunt & 181 & $15-25$ \\
- & - & & - \\
89 & Shunt & 94 & $895-885$ \\
1 & Series & 1 & $1825-1815$ \\
2 & Series & 2 & $1815-1805$ \\
- & - & & - \\
89 & Series & 89 & $945-935$ \\
\hline \hline
\end{tabular}

\subsection{FAULT IDENTIFICATION FOR ANALOG MODEL}

The impulse current signals obtained under series and shunt faults as well as no-fault condition of the analog model of the 3 MVA transformer have been de-noised with the help of wavelet packet as discussed in an earlier section. Then the data for SVM for analog model of the transformer has been organized following the same procedure as discussed for the digital model, with the dimension of feature vector to be 4 .

\subsubsection{TRAINING PROCEDURE AND SELECTION OF SVM PARAMETERS}

In order to classify these input sets with the help of SVM, training of SVM has been carried out. In this case the SVM has been trained with the input sets obtained under all fault as well as no-fault condition for the EMTP based digital model. After training, all the input sets obtained under fault as well as no-fault condition of the analog transformer have been presented to the SVM for predicting the fault classes.

\subsubsection{RESULTS AND OBSERVATION}

Figure 10 summarizes the results of fault pattern identification performed by the SVM using the analog denoised data. The results clearly show that the SVM trained with simulated data only was capable of identifying the faults recorded experimentally from the analog model with a tolerance limit of $\pm 3.37 \%$. The level of accuracy was thus found to be reasonably good. 
In order to evaluate the performance of the wavelet packet based filter as discussed in earlier section, the impulse current signals obtained under series and shunt faults as well as nofault condition of the analog model of the 3 MVA transformer have been used for predicting the fault location in the same manner. Figure 11 summarizes the results of fault pattern identification performed by the SVM using the raw analog data. The tolerance limit in this case is found to be $\pm 8.01 \%$. The obtained result shows that the de-noising is necessary for proper prediction of fault location.

\section{IDENTIFICATION OF DYNAMIC FAULT}

In order to identify dynamic series and shunt faults, data have been generated as discussed in section 3.2 for the digital model of 3 MVA transformer with the application of time dependent switch. Then the fractal features have been calculated and consequently a feature vector has been formed as in the case of static fault identification for the digital model, the procedure of which has been discussed in section 7 .

For the training of SVM the procedure is same as that in the case of static series and shunt fault identification for digital model of 3 MVA transformer. But assigned target value ranges have been chosen differently so that dynamic faults can be separately identified in addition to the static series and shunt type faults. In this case target value ranges $(\mathrm{T})$ are based on the linear function

$$
T=-10 x+3780+R(5)
$$

The values of other parameters of SVM have been kept same. After training, feature vectors except those which were used for training were presented to SVM for prediction of fault classes. The SVM predicted results for dynamic faults have been plotted in Figure 12. It is seen that the SVM engine has been successful in identifying dynamic faults. Prediction of dynamic shunt faults was found to be more accurate as compared to series faults. The tolerance for location of dynamic faults is calculated to be as small as $\pm 2.85 \%$.
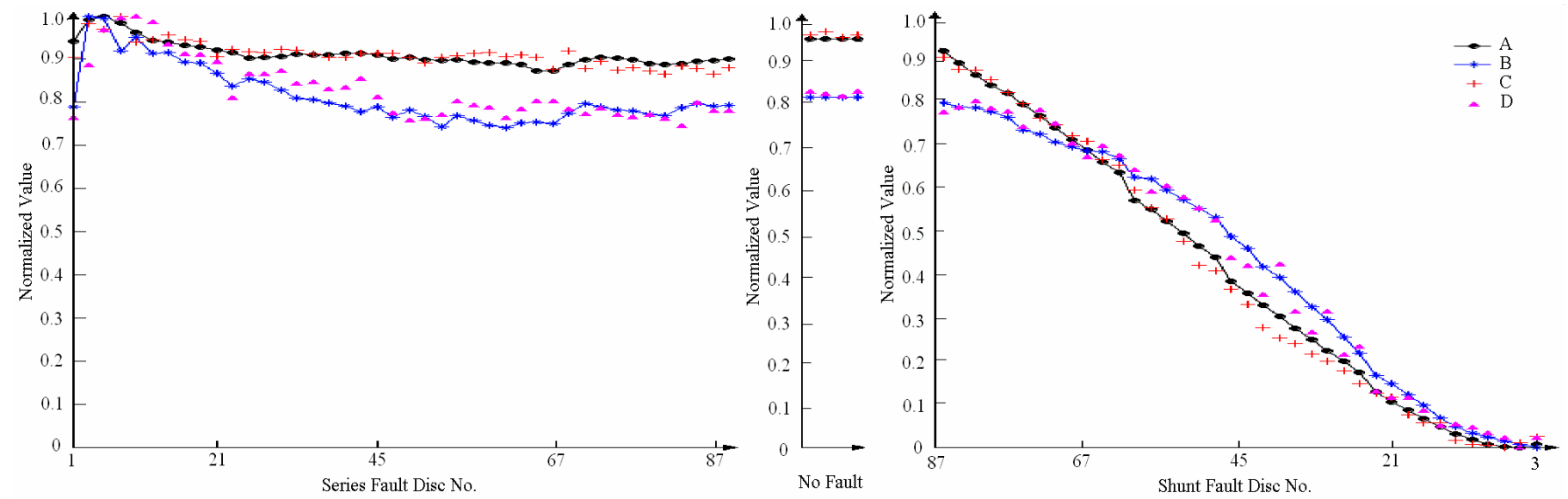

Figure 7. Normalized values of FD using box count and Higuchi's algorithm vs. Fault location. (A) FD using Higuchi's algorithm for impulse response of digital model (B) FD using box count algorithm for impulse response of digital model (C) FD using Higuchi's algorithm for impulse response of analog model (D) FD using box count algorithm for impulse response of analog model.
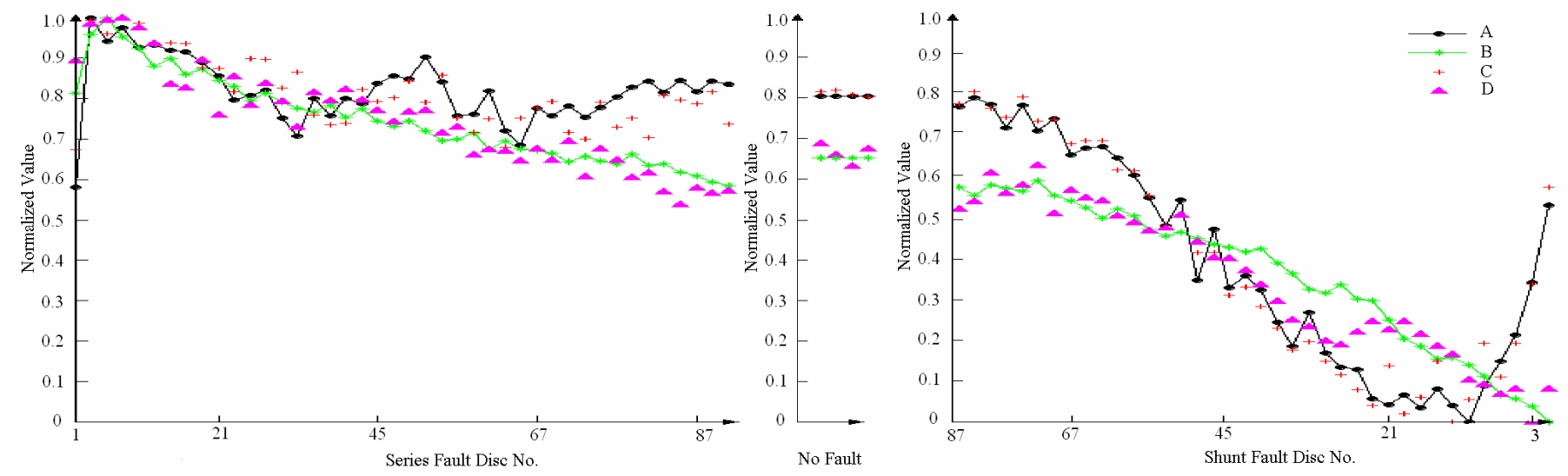

Figure 8. Normalized values of Lacunarity and ApEn vs. Fault location. (A) Lacunarity for impulse response of digital model (B) ApEn for impulse response of digital model (C) Lacunarity for impulse response of analog model (D) ApEn for impulse response of analog model. 

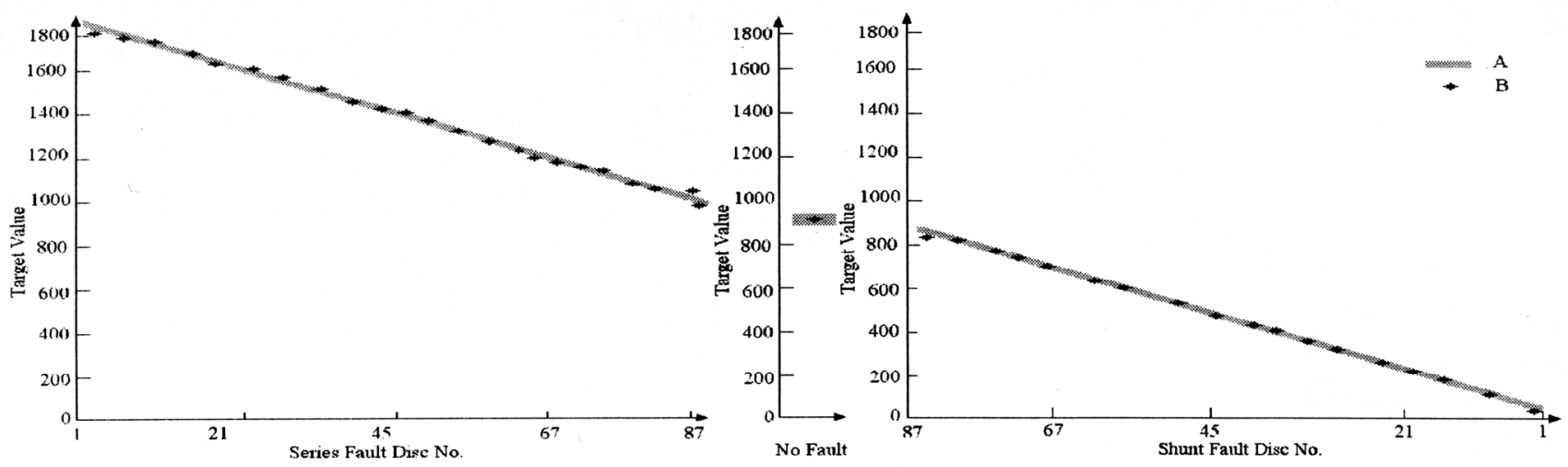

Figure 9. Target value ranges and SVM predicted value vs. static faults at different disc of digital model of 3 MVA transformer. Plot (A) Target value (B) SVM predicted value.
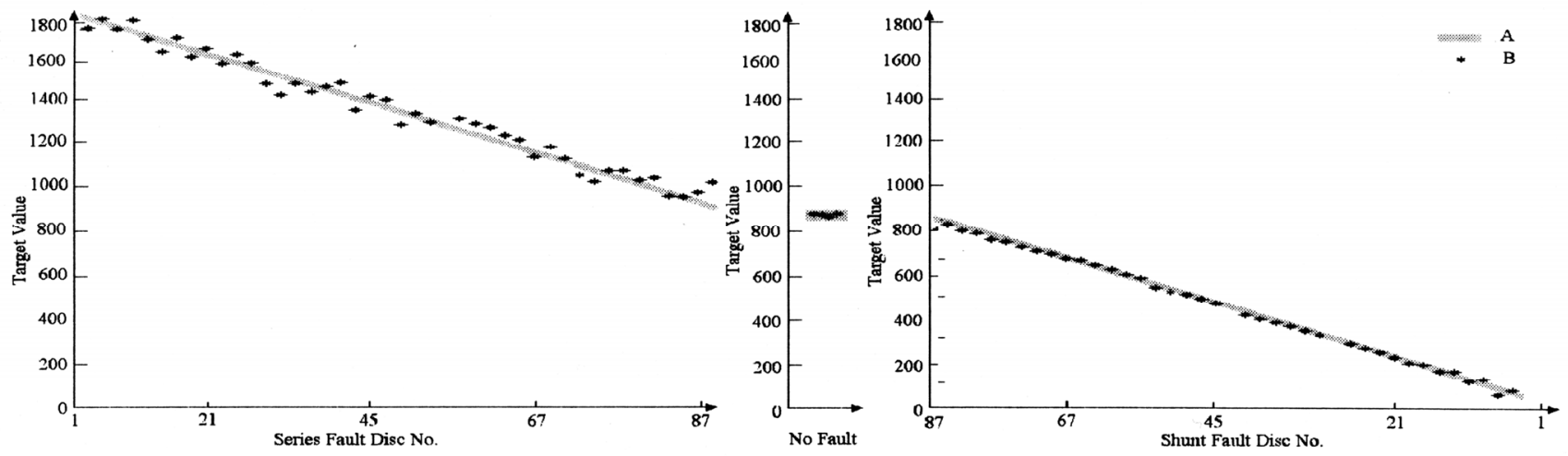

Figure 10: Target value ranges and SVM predicted value (using analog de-noised data) vs. static faults at different disc of analog model of 3 MVA transformer. Plot (A) Target value (B) SVM predicted value.
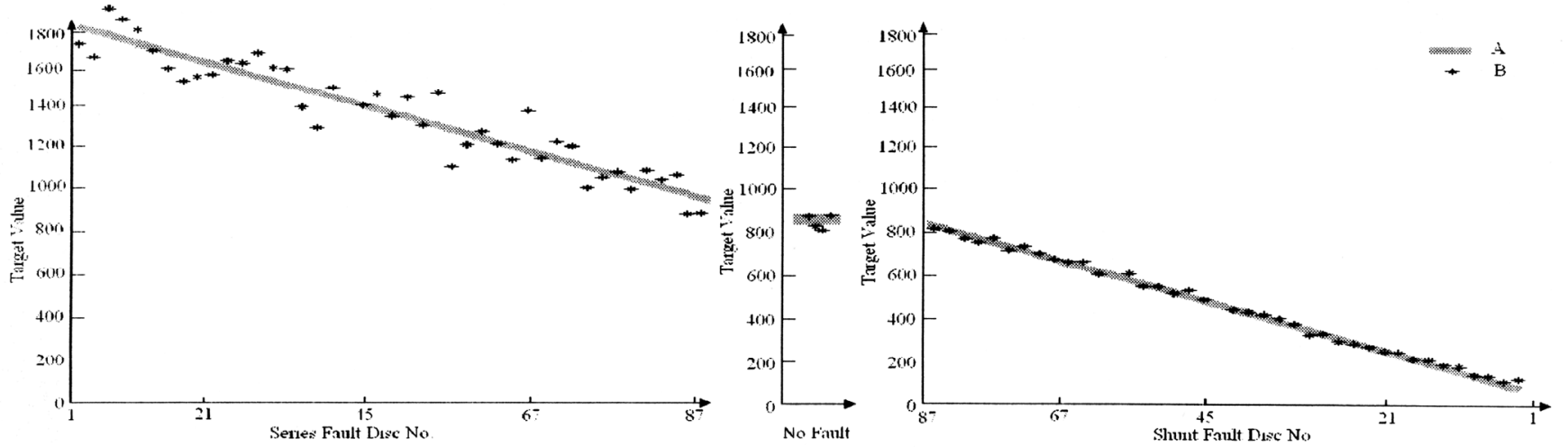

Figure 11. Target value ranges and SVM predicted value (using raw analog data containing noise) vs. static faults at different disc of analog model of 3 MVA transformer. Plot (A) Target value (B) SVM predicted value.
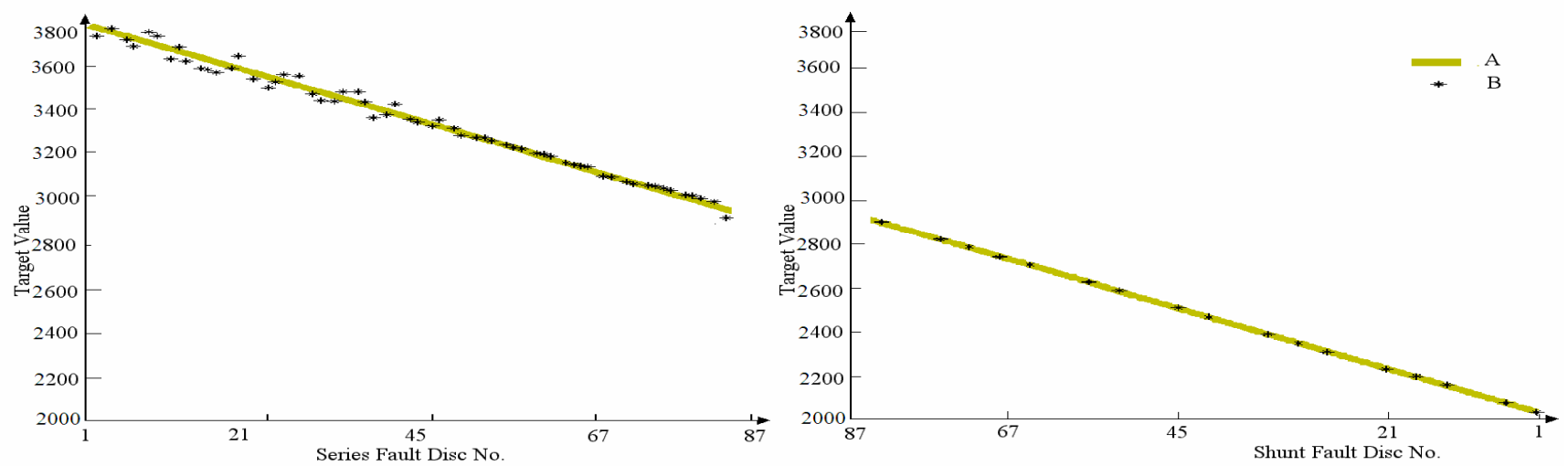

Figure 12. Target value ranges and SVM predicted value vs. dynamic faults at different disc of digital model of 3 MVA transformer. Plot (A) Target value (B) SVM predicted value. 


\section{CONCLUSIONS}

This paper reports an accurate method to identify and find the exact location of different types of impulse faults in transformers using fractal based feature extraction and SVM based feature classification. Four fractal features were found to be essential for the accurate feature classification. For analog signals, denoising by wavelet packet filtering helps further in fault classification in an unambiguous manner. A notable finding is that the SVM tool trained with only simulated data was found to be capable of predicting fault classes accurately when the analog data were presented to the trained SVM for fault prediction. This finding can be of real interest for the transformer manufacturers. The digital model can be developed following standard procedures from the design parameters and the physical dimension of the transformer. Then one can identify faults developed in a real transformer during impulse test by simply training the SVM tool with digital simulated data.

It has been also observed that the algorithm used to identify static faults is also capable of identifying dynamic faults with acceptable accuracy.

\section{REFERENCES}

[1] "Guide to the Lightning and Switching Impulse Testing of Power Transformers and Reactors", IEC Standard, Publication 60076-4, 2002.

[2] E. Stenkvist, "Study of Fault Detection and Failure Location During Surge Testing of Transformers", CIGRE 1952, Supplement to paper no. 12-125.

[3] R. Malewski and B. Poulin, "Impulse testing of power transformers using the transfer function method", IEEE Trans. Power Delivery, Vol.3, pp.476-489, 1998.

[4] P. Purkait and S. Chakravorti, "Time and frequency domain analyses based expert system for impulse fault diagnosis in transformers", IEEE Trans. Electr. Insul., Vol.9, pp. 433-445, 2002.

[5] A. De and N. Chatterjee, "Recognition of impulse fault patterns in transformers using Kohonen's self-organizing feature map", IEEE Trans. Power Delivery, Vol.17, pp.489-494, 2002.

[6] P. Purkait and S. Chakravorti, "Pattern classification of impulse faults in transformers by wavelet analysis", IEEE Trans. Electr. Insul., Vol. 9, pp.555-561, 2002.

[7] A. De and N. Chartterjee, "A Fuzzy ARTMAP Fault Classifier for Impulse Testing of Power Transformer”, IEEE Trans. Electr. Insul., Vol. 11, pp. 1026-1036, 2004.

[8] R. Esteller, G. Vachtsevanos, J. Echauz and B. Litt, "A Comparison of Waveform Fractal Dimension Algorithms", IEEE Trans. Circuit and System-I: Fundamental Theory and Applications, Vol. 48, pp. 177-183, 2001.

[9] A. Krivda, E. Gulski, L. Satish and W. S. Zaengl, "The Use of Fractal Features for Recognition of 3-D Discharge Patterns", IEEE Trans. Electr. Insul., Vol. 2, pp. 889-892, 1995.

[10] P. Purkait and S. Chakravorti, "Impulse Fault Classification in Transformers by Fractal Analysis", IEEE Trans. Electr. Insul., Vol. 10, pp. 109-116, 2003.

[11] A. Ganapathiraju, J. H. Hamaker, and J. Picone, "Applications of support vector machines to speech recognition", IEEE Trans. Signal Processing, Vol. 52, pp. 2348-2355, 2004.

[12] J. Millet-Roig, R. Ventura-Galiano, F. J. Chorro-Gasco and A. Cebrian, "Support vector machine for arrhythmia discrimination with wavelet transform-based feature selection", Computers in Cardiology, Cambridge, MA, USA, pp. 407-410, 2000.
[13] C. Koley, P. Purkait and S. Chakravorti, "Time-Frequency Representation of Resistance for Modeling of Transformer Winding under Impulse Test", IEEE Trans. on Power Delivery, 2006, in Press.

[14] C. K. Roy and J. R. Biswas, "Studies on Impulse Behavior of a Transformer Winding With Simulated Faults by Analogue Modelling", IEE Proc.-C, Vol. 141, pp. 401-412, 1994.

[15] M.V. Wickerhauser, Adapted Wavelet Analysis from Theory to Software, New York: IEEE Press, pp. 237-272 and 443-462, 1994.

[16] C. Koley, P. Purkait and S. Chakravorti, "Wavelet Aided SVM Tool for Impulse Fault Identification in Transformers" IEEE Trans. Power Delivery, TPWRD-00050-2005.

[17] B. B. Mandelbrot, The Fractal Geometry of Nature, W. H. Freeman and Company, New York, 1983.

[18] K. L. Wong, "Application of Very-high-Frequency (VHF) Method to Ceramic Insulators", IEEE Trans. Electr. Insul., Vol. 11, pp. 1057-1064, 2004.

[19] R .F. Voss, "Random Fractals: Characterisation and measurement", in Scaling Phenomena in Disordered Systems, eds. Roger Pynn and Arne Skjeltrop, Plenum press, New York, pp. 1-11, 1985.

[20] K. K. L. Ho, G. B. Moody, C. K. Peng, J. E. Mietus, M. G. Larson, D. Levy and A. L. Goldberger, "Predicting survival in heart failure case and control subjects by use of fully automated methods for deriving nonlinear and conventional indices of heart rate dynamics", Circulation, Vol. 96, pp. 842-848, 1997.

[21] S. M. Pincus, "Approximate entropy as a measure of system complexity", Proc Natl Acad Sci USA, Vol. 88, pp. 2297-2301, 1991.

[22] V. N. Vapnik, Statistical Learning Theory, New York: Wiley, 1998.

[23] N. Cristianini and J. Shawe-Taylor, Support Vector Machines, Cambridge University Press, 2000.

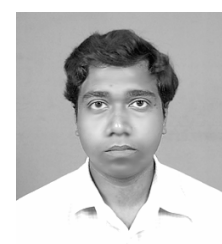

Chiranjib Koley (M'03) was born in Tarakeswar, India in 1978. He obtained the B.Tech degree from HIT, Haldia and the M.Tech degree from IIT, Delhi, India in 2000 and 2002, respectively. Presently he holds the post of Lecturer in the Department of Electronics and Instrumentation Engineering, Haldia Institute of Technology, Haldia, India. His current research includes advanced signal processing applications in High Voltage Engineering.

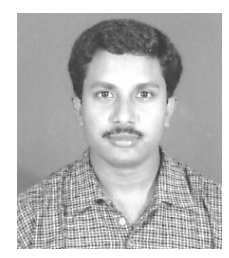

Prithwiraj Purkait (M'97) was born in Kolkata, India in 1973. He obtained the B.E.E., M.E.E. and Ph.D. degrees from Jadavpur University, Kolkata, India in 1996, 1999 and 2002, respectively. He worked with M/s Crompton Greaves Ltd, Mumbai, India as a Design Engineer for one year. He was involved in post-doctoral research in the University of Queensland, Australia during 2002-2003. Presently he holds the post of Assistant Professor and Head, Department of Electrical Engineering Haldia Institute of Technology, Haldia, India. His current research includes transformer insulation condition assessment techniques and advanced signal processing applications in High Voltage Engineering.

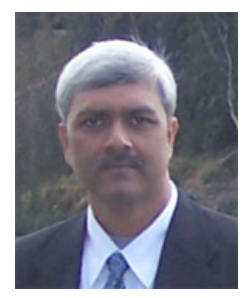

Sivaji Chakravorti (M'89-SM'00) was born in Kolkata, India in 1962. He obtained the B.E.E, M.E.E and Ph.D. degrees from Jadavpur University, Kolkata in 1983, 1985 and 1993, respectively. In 1994 he did his post-doctoral research at Indian Institute of Science, Bangalore, India. He worked at the Technical University, Munich as Alexander von Humboldt Foundation research fellow during 1995-96 and also in 1999. Since 1985 he is a faculty member of EE dept. JU., where he is currently Professor. In 1998, he worked with Siemens AG, Berlin as Development Engineer. His current research interests are condition monitoring of large electrical equipment, modeling of partial discharge, high voltage system optimization and virtual laboratory techniques. 